\title{
FISICA ARISTOTELICA \\ E ONNIPOTENZA DI DIO IN GUGLIELMO DI OCKHAM
}

Alessandro Ghisalberti

SÍNTESE - A Física aristotélica é questionada em diversos de seus princípios por autores dos séculos XIII e XIV, embora eles ainda a aceitem enquanto todo. Ao tratar do problema do poder absoluto de Deus, Ockham afasta-se tanto do "princípio de plenitude" do mundo neoplatônico, como também do "mundo fechado" de Aristóteles.
ABSTRACT - The Aristotelian Physics is controverted in not few of its principles by authors of the thirteenth and fourteenth century, although they accept it as a whole. Dealing with the problem of God's absolute power, Ockham deviates from the Neoplatonic "principle of fullness" of the world as well as from Aristotle's "closed cosmos".

\section{1 - Aristotele e le condanne parigine del 1277}

L'intervento con cui il vescovo di Parigi, Stefano Tempier, il 7 marzo del 1277, sottopose al vaglio dell'ortodossia cristiana 219 proposizioni prevalentemente ricavate da testi di metafisica e di fisica di Aristotele o dei suoi interpreti arabi e latini ha segnato una svolta estremamente importante nell'atteggiamento dei maestri latini dell'età successiva. Il sillabo di Tempier presenta una lunga serie di enunciati che, sottoposti a un rigoroso vaglio logico-linguistico, mostrano punti di attrito con l'insegnamento biblico o con la formulazione tradizionale degli articoli del credo cristiano; essi perciò devono essere riformulati, quando l'incompatibilità col dogma fosse solo di carattere formale, o respinti, quando veicolano una dottrina intrinsecamente in contrasto con la verità rivelata o con suoi corollari diretti.

Alcuni di questi articoli del Sillabo si collegano al problema della creazione, in quanto mirano a respingere ogni prospettiva dottrinale che finisca con porre dei vincoli all'onnipotenza di Dio, uno dei primi attributi divini professati dal credo cattolico: "Credo in unum Deum, patrem omnipotentem, creatorem caeli et terrae".

Cosi l'art. 34 di Tempier censura la seguente proposizione: "Che la causa prima non può produrre una pluralità di mondi"; l'art. 49 considera inaccettabili le implicazioni concernenti questa dottrina: "Che Dio non può muovere il cielo con un movimento rettilineo, per la ragione che lascerebbe un vuoto". L'impossibilità dell'esistenza del vuoto è all'origine anche dell'art. 201: "Che colui che genera il mondo nella sua totalità pone ił vuoto, in quanto il luogo precede necessariamente ciò che

\begin{tabular}{|l|l|l|l|l|l|}
\hline VERITAS & Porto Alegre & v. 41 & $\mathrm{n}^{0} 163$ & Setembro 1996 & p. $483-492$ \\
\hline
\end{tabular}


viene generato nel luogo; quindi prima della generazione del mondo sarebbe esistito un luogo senza un corpo collocato, ossia il vuoto".

Un ultimo richiamo, che ritengo utile a introdurre la problematica cosmologica, riguarda l'articolo 147: "Quod impossibile simpliciter non potest fieri a Deo, vel ab agente alio. Error, si de impossibili secundum naturam intelligatur", ${ }^{1}$ ossia la proposizione che Dio o un altro agente non possono produrre ciò che è impossibile in assoluto è erronea se non si distingue tra impossibile in sé e impossibile secondo natura; mentre l'impossibile in sé non può essere prodotto né da Dio, né da altre potenze naturali, ciò che si configura come impossible secondo natura, ossia ciò che risulta in contrasto con qualche legge naturale, può essere prodotto da Dio, in forza della sovrana potenza creatrice. Viene spontaneo, a questo proposito il richiamo alla distinzione di Guglielmo di Ockham fra potentia Dei absoluta e potentia Dei ordinata: la prima riguarda tutto ciò che Dio può sovranamente compiere, e l'assolutezza (absoluta) del suo volere non ha altri limiti che l'intrinsecamente contraddittorio; la potenza ordinata verte sugli interventi possibili a Dio in riferimento all'ordine del cosmo da lui stesso stabilito. Il Venerabilis Inceptor precisa: "Questa distinzione non va intesa come se si ponessero in Dio due potenze realmente distinte, di cui l'una è ordinata e l'altra assoluta, dal momento che in Dio c'è un'unica potenza che riguarda il suo agire ad extra, e tale potenza da ogni punto di vista coincide con Dio stesso. La distinzione non va intensa nemmeno come se si dicesse che Dio può fare aicune cose ordinatamente, mentre può fare altre cose assolutamente e in modo non ordinato, poiché Dio non può fare nulla in modo non ordinato. La distinzione va intensa cosi: "potere qualcosa" talvolta viene preso facendo riferimento alle leggi ordinate e istituite da Dio; si dice allora che Dio può fare quelle cose in base alla potenza ordinata. Altre volte "potere" viene preso facendo riferimento a tutto ciò che non include contraddizione essere prodotto, sia che Dio abbia stabilito di produrre in seguito quelle cose, sia che non l'abbia stabilito, dal momento che Dio può fare molte cose che pure non vuole fare, secondo il parere di Pietro Lombardo (Libri Sententarium, I, d. 43): in riferimento a queste cose si dice che Dio le può produrre in base alla potenza assoluta". ${ }^{2}$ Questo lucido passo del sesto Quodlibet ci consente di entrare subito nel ruolo centrale circa il tema della creazione così come è stato dibattuto dopo il 1277, in particolare da Giovanni Duns Scoto e dallo stesso Ockham: il "potere" del Dio onnipotente non è rivendicato solo come dominio dell'ordine da lui creato, o come affermazione della trascendenza divina pura e immutabile rispetto al mondo diveniente e caduco; si analizza con rigore logico anche l'immenso ordine del possibile, ossia del positivamente non contraddittorio, rapportando la volontà divina al suo "potere" circa gli atti creativi o distruttivi, circa le azioni dirette o le azioni mediate, che si configurano como possibili in quanto non implicano nulla che sia contraddittorio farsi. Si capisce come in

1 Gli articoli del Sillabo sono pubblicati da H. DENIFLE - A. CHATELAIN, Chartularium Universitatis Parisiensis, tomo I, Parigi 1899, p. 543-558. Due studi sono fondamentali per l'analisi del documento: R. HISSETTE, Enquête sur les 219 articles condamnés à Paris le 7 mais 1277, Louvain-Paris 1977; L. BIANCHI, II vescovo e i filosofi. La condanna parigina del 1277 e l'evoluzione dell'aristotelismo scolastico, Bergamo 1990.

2 OCKHAM, Quodlibet VI, q. 1, art. 1; Opera theologica IX, p. 585-586. Traduzione italiana in Guglielmo di Ockham, Scritti filosofici, a cura di A. Ghisalberti, Firenze 1991, p. 196-197. 
questo dibattito la creazione non diventi più riferimento solo a ciò che Dio ha fatto o fa storicamente, ma anche a tutto ciò che farà, a tutto ciò che può fare, anche se Dio non è tenuto a fare tutto ciò che può fare, e perciò si deve dire che ci sono delle cose ("creazioni") possibili, che pure non passeranno mai all'atto.

Se l'ambito del dibattito scotista e ockhamista circa la creazione è ben inquadrato dalla distinzione fra la potenza assoluta e ordinata in Dio, va però subito notato che non riteniamo fondata l'equazione diretta tra la distinzione delle due potenze in Dio e la teologia cosidetta "nominalistica", che accentuerebbe il momento arbitraristico del volere in Dio o, quanto meno, si configurerebbe come una teologia della salvezza totalmente dipendente dall'insondabile volere divino, anticipazione della concezione luterana di Dio. Non è consentito dai testi dei nostri autori asserire che l'insistenza sull'onnipotenza divina sia volta a toglieri valore allo studio delle leggi naturali, né che insinui dubbi circa la capacità conoscitiva dell'uomo nei confronti del mondo oggetivo: come risulterà dall'analisi delle singole posizioni, il metodo di indagine è totalmente fiducioso nelle capacità della ragione, essendo legato al rigore logico sia nell'esegesi dei testi scritturistici, sia nell'estensione delle inferenze possibili a partire da ciò che la rivelazione insegna in modo inequivocabile.

L'osservazione metodologica che stiamo avanzando va completata mediante un'altra considerazione, di tipo storico: la distinzione fra la potenza asoluta e ordinata in Dio, come in generale l'inserimento del dogma dell'onnipotenza nelle questioni teologiche sulla creazione, non è stata una novità di Ockham, né di Duns Scoto; troviamo la distinzione, intorno al 1230, nel domenicano Ugo di St. Cher, nella Summa theologica di Alessandro di Hales, in molte opere di Tommaso d'Aquino, con particolare isistenza nel Commento alle Sentenze, e nella Summa theologiae di Alberto Magno; anzi, è proprio Alberto Magno il primo a đichiarare che si tratta di una distinzione di uso comune (dici consuevit). ${ }^{3}$ Una dottrina comumente accolta dai teologi scolastici diventa il punto discriminante, per come essa viene applicata nella trattazione delle questioni, il luogo di emergere di atteggiamenti diversi, di prospettive teologiche diversificate. E per scrupolo di storico, aggiungo anche che il documento censorio del 1277 non fu esplicitamente costruito in modo da opporre alla concezione necessitaristica del mondo greco-arabo la dottrina specifica della potentia Dei absoluta, ma difendeva semplicemente la libertà dell'agire divino da ogni sorta di limitazione. E'stata la riconsiderazione globale degli attributi divini e delle leggi cosmologiche, esigita dalle condanne del Sillabo, il quale in particolare chiedeva il superamento della lettura di Aristotele accolta dai seguaci dell'averroismo latino, a condurre a una maggiore presa di coscienza della radicale differenza tra gli attributi del Dio biblico, onnipotente creatore, e il primo motore immobile del mondo, della tradizione peripatetica. Lo slittamento nel modo di intendere l'onnipotenza divina, da semplice attributo che si rispecchia nel meraviglioso ordine del creato (sec. XIII), alla facoltà divina di produrre, senza alcun mediatore, tutto il producibile (prima metà del sec. XIV) ha trovato il suo movente principale nella revisione radicale della lettura della metafisica e della cosmologia aristotelica.

3 Cfr. L. MOONAN, St. Thomas Aquinas on divine power, in "Atti" del Congresso Intemazionale Tommaso d'Aquino nel settimo centenario, vol. III, Napoli 1976, p. 366-407; E. RANDI, Il sovrano e l'orologiaio. Due immagini di Dio nel dibattito sulla "potentia absoluta" fra XIII e XIV secolo, Firenze 1977. p. 18-33. 


\section{2 - Onnipotenza divina e contingenza del mondo: da Duns Scoto a Ockham}

E' sicuramente assai stimolante l'analisi delle implicazioni dell'onnipotenza divina a livello teologico e a livello filosofico che sia Duns Scoto, sia Ockham sviluppano in primo luogo commentando il primo libro delle Sentenze di Pier Lombardo, il quale alle distinzioni 42-43-44 aveva raccolto il materiale relativo all'onnipotenza divina: qual è il potere di Dio, raffrontandolo alla nozione umana di possibile; come si possono mantenere sia l'immutabilità sia l'onnipotenza in Dio; quale il rapporto tra l'esistente e la possibilità connessa con la libertà del creatore. ${ }^{4}$ Duns Scoto introduce l'esposizione del testo domandandosi se l'onnipotenza divina possa essere dimostrata con la regione naturale, e risponde con una distinzione che molto ci i1lumina circa lo slittamento della problematica di cui sopra dicevamo: ocorre distinguere tra la nozione filosofica di onnipotenza e quella teologica. Si può dire che Dio è onnipotente in quanto è la prima causa efficiente dotata di potenza attiva, che si estende a ogni effetto in quanto causa prossima o in quanto causa remota; l'onnipotenza così intesa di sua natura ammette il concorso attivo delle cause seconde, ed è un attributo razionalmente dimostrabile del "primum efficiens". Diversa è invece l'accezione teologica, razionalmente indimostrabile, la quale denota che Dio può produrre tutte le cose possibili senza il ricorso alle cause intermedie, con l'intervento diretto e immediato della sua sola potenza. Per poter dimostrare razionalmente questo attributo dovremmo poter rigorosamente provare la seguente proposizione: la prima causa effetiva può fare direttamente ciò che può fare mediante una causa seconda; ma l'ordinamento delle cause seconde non permette di giungere a questa affermazione, perché "anche se il sole possedesse una causalità più eminente di un bue o di un altro animale, non per questo si potrebbe concedere che il sole può generare immediatamente un bue così come può farlo attraverso il bue-causa". ${ }^{5}$ Da un punto di vista filosofico è problematica l'idea che Dio possa svolgere la funzione delle cause seconde; le cause seconde sono tali constitutivamente per il loro relazionarsi alla causa prima, e quindi, nella prospettiva comune ai pensatori del secolo XIII, l'ipotesi dell'abolizione della causalità seconda induce all'abolizione della primalità della causalità prima. Come ben si esprimerà lo scotista Francesco di Meyronnes, un conto è che Dio possa produrre un "raggio", un altro è che Dio possa produrre un "raggio di sole", che è tale proprio perché proviene dal sole. ${ }^{6} \mathrm{~L}$ esclusione dell'intervento diretto e immediato di Dio, precisa Duns Scoto, non è per innalzare la perfezione della causalità seconda, bensì perché l'imperfezione degli effetti delle cause seconde è tale da rendere ripugnante la loro attribuzione alla potenza immediata di Dio; l'ultimum cui può giungere la ratio naturalis è che la prima

4 Cfr. PIETRO LOMBRADO, Sententiae, I, dist. 42-44; vol. I, parte I, Firenze 1972, p. 294-306.

5 GIOVANNI DUNS SCOTO, Ordinatio I, dist. 42, q. unica; ed. Vaticana, VI, p. 344.

6 "Ponitur exemplum de radio solis habente respectum generationis passivae ad solem. Si autem fieret a Deo, esset quidem radius, sed non haberet respectum generationis passivae ad solem, ita quod sol terminaret illum respectum, ita quo diceremus quod esset a sole" (FRANCESCO DI MEYRONNES, In I Sent., prol., 28; ed. Venezia 1520, f. 10 P-O. Cit. da L. BIANCHI, Il vescovo e $i$ filosofi, cit., p. 97. nota 97). 
causa efficiente possiede una potenza effetiva più eminente della potenza di qualsiasi altra causa effettiva, ed anche che possiede in modo eminente la potenza effettiva di qualsiasi altra causa. ${ }^{7}$ L'onnipotenza che Scoto chiama teologia configura invece il cosiddetto "postulato dell'immediatezza", ossia che la causalità divina possa sostituirsi integralmente alla causalità finita, possa cioè ignorare le condizioni causali, possa sconvolgerle e fare direttamente tutto ciò che può fare con le cause seconde ed anche tutto ciò che postulerebbe un diverso ordine delle cause seconde. L'esegesi scotista dell'onnipotenza teologica viene sviluppata e ampliata da Ockham, il quale deve perciò confrontarsi con le censure del Tempier, e precisamente con l'articolo 69: "Ouod Deus non potest in effectum causae secundariae sine ipsa causa secundaria". Il riferimento all'onnipotenza come capacità di fare tutto il possibile presente nell'enunciato di Tempier è maturato in una nuova proposizione: Dio può fare tutto il possibile liberamente e senza alcuna mediazione, in cui si esprime il ricordato principio di immediatezza, da Ockham chiamato "propositio famosa theologorum. ${ }^{8}$

Il Venerabilis Inceptor, commentando la distinzione 42 del primo libro delle Sentenze di Pier Lombardo, esordisce chiedendosi se Dio possa fare tutto ciò che può essere fatto dalle creature; assume cioè come unica nozione valida di onnipotenza quella che Duns Scoto qualificava come nozione teologica, e che includeva la possibilità per Dio di fare ogni cosa possibile alla creatura anche senza la cooperazione della creatura. Ockham risponde che la causalità totale e diretta di Dio non è mostrabile, anche perché non si può rigorosamente dimostrare che Dio sia di fatto l'unica causa di tutte le cose, e nemmeno che egli causi in modo libero e contingente: se non si può dimonstrare apoditticamente, ma solo con degli argomenti probabili, la creazione dal nulla, tanto meno si potrà provare razionalmente che Dio può "omnia immediate et se solo, sine omni causa concausante, causare". ${ }^{9}$ L'analisi del concetto di creazione dal nulla è svolta nel Commento al secondo libro delle Sentenze, dove Ockham spiega le ragioni della indimostrabilità razionale dell'affermazione che Dio agisce liberamente e senza alcun mediatore. In particolare egli sottolinea come nei testi dei filosofi pagani non compaia mai la tesi che Dio sia, in tutto e per tutto, causa omnium. Si può giungere a dire che per Aristotele Dio è causa efficiente di tutte le cose, dal momento che lo Stagirita sostiene che Dio è la causa finale di tutte le realtà, che si muovono in quanto sono attratte finalisticamente da Dio, e la causa finale, per essere veramente tale, deve essere anche causa efficiente. Ma non si può assolutamente dire che Aristotele abbia posto la causalità

7 "Et hoc modo videtur omnipotentia esse credita de primo efficiente, et non demonstrata, quia licet primum efficiens habeat in se potentiam effectivam eminentiorem potentia cuiuscumque alterius causae effectivae, habeat etiam in se eminenter potentiam effectivam cuiuscumque alterius causae (sicut deductum est distinctione 2 et per hoc probatum est eum habere potentiam infinitam) et istud sit quasi ultimum ad quo ratio naturalis possit attingere, de Deo cognoscendum, tamen ex hoc non videtur concludi omnipotentia secundum intellectum" (GIOVANNI DUNS SCOTO, Ordinatio, I, d. 42, q. unica; ed. Vaticana, VI, p. 343).

OCKHAM, Quodlibet VI, q. 6, concl.; Opera theologica, IX, p. 604. Per le riserve di Okcham circa la dottrina scotista dell'onnipotenza, cfr. A. GHSALBERTI, Onnipotenza divina e contigenza del mondo in Guglielmo di Ockham, in AA. VV., Sopra la volta del mondo. Onnipotenza e potenza assoluta di Dio tra medioevo e età moderna, Bergamo 1986, p. 34-55.

OCKHAM, In I Sent., d. 42, q. unica; Opera theologica, IV, p. 617. 
totale del primo motore, pari a quella sottesa dal concetto biblico di creazione: pur essendo causa di tutte le cose, il primo motore aristotelico non causa contingenter, non causa de novo, non causa liberamente. Causare contingenter significa produrre una cosa e non un'altra, significa che Dio nella sua attività creatrice aequaliter respicit plura, assunto questo che non era accettato né da Aristotele, né dai peripatetici arabi; secondo questi filosofi Dio non si rapporta egualmente a tutte le cose producibili, ma produce la prima intelligenza in modo necessario e, mediante la prima, produce la seconda, e così via. Il Dio aristotelico non stabilisce alcun rapporto diretto con le realtà sensibili, non conosce né muove direttamente i corpi sublunari, che agiscono sotto le influenze dei corpi celesti; diversa la concezione cristiana per cui "Dio è causa immediata di qualsiasi cosa producibile, causa totale o parziale, che sviluppa immediatamente la causalità di qualsiasi causa seconda". ${ }^{10}$

\section{3 - Possibilità di un mondo migliore: Ockham contro Aristotele}

Nella distinzione 43 del commento al primo libro delle Sentenze troviamo un approfondimento: "Utrum Deus possit facere aliqua quae non facit nec faciet"; Ockham si interroga cioè se si possa dimostrare la libertà della volontà divina, in modo che risulti dimostrata anche la sua onnipotenza como facoltà di fare altro da ciò che compie. La risposta del Venerabilis Inceptor ripropone la convinzione che abbiamo anticipato: non si può dimostrare apoditticamente la libertà della volontà divina, anche perché l'esistenza della volontà libera non si dimonstra, mas si desume dall'esperienza e l'esperienza ci consente di dire che Dio è un soggetto volente, ma non che è anche volente libero: Dio esula infatti da ogni approssimazione empirica. Non si può dimostrare la libertà, dunque non si può dimostrare l'onnipotenza; questo non significa che sia dimostrabile la tesi negativa: ammettere l'onnipotenza divina non è affatto contraddittorio, anzi la negazione dell'onnipotenza configurerebe l'assurda tesi che l'azione creatrice di Dio è quella di una causa naturale: "Sed si esset causa naturalis, vel omnia produceret simul vel'nulla, quorum utrumque patet esse manifeste falsum. Et ideo manifeste falsum est Deum esse causam naturalem aliorum a se". ${ }^{11}$

La distinzione 44 sviluppa le conseguenze della non contraddittorietà della radicale libertà del volere divino, del suo poter agire contingenter, facendo cose diverse da quelle che fa: Dio può creare un mondo migliore del nostro?

Ockham premette una precisazione circa il significato del termine "mundus": per mondo si deve intendere non tanto tutto ciò che Dio ha creato, ma l'insieme composto dalla moltitudine delle sostanze contenute in un unico corpo; più il cor-

10 OCKHAM, In II Sent., q. 5; Opera Theologica V. p. 87. Ockham è molto attento a distinguere la vera posizione di Aristotele dalle contaminazioni neoplatoniche, in particolare, dall'inserzione nella teologia aristotelica della rigida gerarchia delle sostanze spirituali e celesti compiuta da Avicena; pertanto egli dichiara che Aristotele non ha sostenuto tali vincoli gerarchici per il primo motore, ma l'ha inteso come causa immediata e totale delle sostanze separate e dei corpi celesti incorruttibili; queste sostanze e questi corpi celesti sono a loro volta causa immediata delle sostanze corruttibili, ossia delle generazioni e delle corruzioni. Cfr. A. GHISALBERTI, Onnipotenza divina e creazione dal nulla in Guglielmo di Ockham, ultimo capitolo del volume Medioevo teologico. Categorie della teologia razionale nel Medioevo, Roma-Bari 1990, p. 147-162.

11 Ockham, In I Sent., d. 43, q. 1; Opera teologica, IV, p. 636. 
po contenente stesso. ${ }^{12} \mathrm{E}^{\prime}$ facile riconoscere in questa definizione la descrizione aristotelica del cosmo, un'unica sfera immensa ma finita, composta dalle sostanze effettivamente esistenti, che sono anche le uniche sostanze possibili per Aristotele, il cui limite esterno è costituito dal cielo delle stelle fisse e che non compatisce il vuoto.

Una seconda precisazione verte sul significato del termine migliore (un mondo "migliore" del nostro): una cosa può essere detta migliore di un'altra in due sensi: riguardo all'essenza o riguardo all'accidente. Nel primo caso indichiamo che una specie è più perfetta di un'altra, nel secondo caso la distinzione riguarda solo un grado di bontà maggiore a parità di specie.

Ciò posto Ockham si chiede se Dio possa creare un mondo migliore sia per essenza, sia riguardo all'accidente. ${ }^{13}$ Tale domanda era già presente in Tommaso, il quale argomentava cosi: una cosa non può essere migliorata nella specie, pena il suo divenire qualcosa d'altro, come il numero 4 non può essere reso maggiore, senza che divenga un numero diverso. Dio può però creare altre cose oltre quelle esistenti, di specie diverse e migliori: quindi anche un mondo più perfetto per essenza, che andrebbe distinto dal nostro. E aggiunge: se Dio aumentasse la bontà del cosmo attuale per essenza, ponendovi nuove specie, darebbe origine a un mondo numericamente distinto da quello in cui viviamo. ${ }^{14} \mathrm{E}$ ' evidente che in questo modo Tommaso pone le basi per ammettere la possibilità per Dio di creare una pluralità di cosmi, dato che il dogma dell'onnipotenza e della libertà divina lo spinge a asserire che il Creatore può, se vuole, rendere più perfetto sotto ogni aspetto ciò che fa. Ma l'Aquinate non sviluppa tale apertura teoretica, perché resta convinto assertore dell'unicità del mondo, cui si rapporta l'unicità del Creatore. ${ }^{15}$. Se Dio migliorasse il mondo per essenza, darebbe origine a un universo diverso dal nostro, ma si avrebbe pur sempre un solo mondo. Per quanto riguarda invece la perfezione secondo l'accidente, il nostro mondo potrebbe migliorare, cosiché ci potrebbe essere un aumento del numero dellle sostanze a parità di specie, oppure le cose potrebbero diveniere più buone secondo la quantidà, e ciononostante si avrebbe pur sempre lo stesso cosmo.

Ockham procede oltre: innanzitutto sostiene che non è contraddittorio ammettere un mondo che sia specificamente migliore del nostro, dato che Dio può creare nuove specie più perfette di quelle esistenti. Come già affermava Agostinho, Dio potrebbe infatti creare un uomo che, a differenza dell'uomo storico, non possa peccare. Un tale uomo sarebbe specificamente diverso dagli uomini attuali, perché ciò

12 "In ista quaestione accipiendus est mundus praecise pro uno universo quasi composito ex partibus quae sunt substantiae, et non secundum quod includit accidentia substantiarum" (OCKHAM, In I Sent., d. 44, q. unica; Opera theologica, IV, p. 651).

13 "Videndum est utrum posset facere mundum meliorem isto bonitate essentiali sive substantiali, et hoc distinctum specie; secundum utrum posset facere mundum meliorem distinctum solo numero; tertium ultrum posset facere mundum meliorem bonitate accidentali" (Ibid., p. 651-652).

14 "Deus melius universum facere potuisset et posset [...] Haec autem melioratio omnium partium vel potest intelligi secundum bonitatem accidentalem, et sic esset talis melioratio a Deo manentibus eisdem partibus et eodem universo; vel secundum bonitatem esentialem, et sic esset Deo possibilis, qui infinitas alias species condere potest. Sed sic non essent eaedem partes, et per consequens nec idem universum" (TOMMASO D'AOUINO, In I Sent., dist. 44, art. 1).

Cfr. TOMMASO D'AOUINO, Summa theologiae, I, q. 47, art. 3. 
che può conveniere a un individuo di una specie, non può ripugnare formalmente a un altro individuo della medesima specie. Ma agli uomini esistenti non ripugna il peccare; quindi un essere senza possibilità di colpa sarebe specificamente diverso. ${ }^{16}$ Spontanea l'obiezione: Gesù non poteva peccare, eppure era un uomo como noi. Risponde Ockham: a Gesù in quanto uomo non ripugnava peccare, ma in quanto Verbo di Dio non poteva avere colpe; è la natura divina che rende assurdo ammettere il peccato nel Cristo, non la sua natura umana. ${ }^{17}$ Quindi, secondo l'insegnamento rivelato, non è contraddittorio, ossia è possibile che Dio crei altre specie; d'altra parte la ragione non ha argomenti per dimostrare l'assurdità di tale assunto, per cui deve essere ammessa la possibilità di un mondo specificamente migliore. ${ }^{18}$ A maggior ragione quindi si deve accettare che Dio potrebbe fare un mondo migliore negli accidenti e secondo la quantità, perché Egli potrebbe creare nuovi individui della medesima specie all'infinito. ${ }^{19}$ Ockham però puntualizza che un cosmo migliore, sia riguardo alla specie, sia riguardo al numero di sostanze, non potrebbe coincidere col nostro, ma dovrebbe esserne distinto: ne deriva l'ammissione della possibilità dell'esistenza di una pluralità di mondi, e quindi della possibilità che Dio crei mondi migliori del nostro.

Con queste affermazioni Ockham si stacca definitivamente da quello che Lovejoy chiama il "principio di pienezza", latente nelle filosofie platoniche e neoplatoniche, per le quali nessuna potenzialità di essere manca la propria realizzazione e perciò l'universo è riempito da ogni possibile tipo di essere. Contemporaneamente egli abbandona il mondo chiuso di Aristotele, macchina perfetta ma senza possibilità di variazione o di sottrazione alle cosiddette "leggi naturali": proiettandosi verso la distinzione, esplicitata nel Quodlibet VI, q. 1, tra potenza assoluta e potenza ordinata in Dio, per avallare la possibilità che Dio possa compiere un atto che non si concilia con l'ordine etico da lui imposto all'uomo (è possibile per Dio salvare un' uomo senza infusione della grazia santificante?), ${ }^{20}$ Ockham mira ad affermare un eccedenza delia volontà volente in Dio rispetto alla sua volontà voluta. Nel momento in cui Dio pone l'ordine del mondo attuale, la sua volontà non sottostà ad alcun altro ordine, legge o volere; il creato mostra il suo vero carattere, di entità costituite dal libero atto d'amore del creatore, entità che non vanno assolutizzate, anche perché non esauriscono tutta la capacità divina ad extra, non intaccano la sua assolutezza, il suo essere ab-soluta, sciolta da ogni vincolo e limitazione.

16 Cfr. OCKHAM, In I Sent., d. 44, q. un.; Opera teologica, IV, p. 652-653.

17 Ibid., p. 653.

18 Ibid., p. 655 .

19 "Deus potest facere mundum meliorem isto, et distinctum solo numero ab isto mundo. Cuius ratio est quia infinita individua eiusdem rationis cum illis quae modo sunt, posset Deo producere. [...] Sed non arctatur ad producendum ea in isto mundo. Igitur posset ea producere extra istum mundum et ex eis facere unum mundum, sicut ex illis quae sunt iam producta fecit hoc mundum "(Ibidem). "Deus potest facere mundum meliorem bonitate accidentali, quia hoc non est nisi facere mundum informatum perfectioribus accidentibus. Sed hoc est possibile, cum Deus illa accidentia possit augere et per consequens facere ea perfectiora" (Ibid., p. 660). 585). 


\section{Conclusione: la crisi del "mondo pieno"}

La creazione è un atto originario di Dio, che, restando egli invariato, termina alla creatura, per la quale si dà una variazione sostanziale, quella del venire ad essere, di entrare in relazione con Dio così da costituirsi come un individuo, finito per la natura e per l'essere, ma totalmente singolare, distinto da ogni altro individuo. Sulla intrinseca individualità del creato insistono, con diversità di approccio, sia Duns Scoto sia Ockham, a partire dall'ammissione che entrambi i maestri fanno della possibilità per l'intelletto umano di disporre della conoscenza intuitiva del singolare. In un mondo non eterno, ma termine di un libero atto di volontà e di amore, non è sostenibile la prevalenza dell'essenzialismo universalistico, ma si postula coerentemente la puntuale conoscibilità del singolo ente concreto: ad esso infatti è rivolta ultimativamente la volontà ponente del creatore. L'individuo attualmente esistente non può essere solo oggetto di esperienza sensibile, ma deve essere parimenti oggetto della conoscenza intellettiva immediata: "l'esistente in quanto esistente si costituisce cosi da un lato come fenomeno noetico immediato; dall'altro lato può essere Terminus di un Creatore contingente perché vuole liberamente solo un siffatto esistente, non cioè un esistente che sia scontato (selbverständliches), come accade in un mondo eterno, bensi un fenomeno da interpellare in modo nuovo". ${ }^{21}$

Possiamo ricondurre la valenza teologica, mediante la dottrina della creazione, della dotrina della conoscenza intuitiva intellettiva presente nei nostri autori a una influenza dell'esperienza francescana, all'amore per le cose, per gli animali e soprettutto per i fratelli predicato e vissuto da Francesco d'Assisi. L'incidenza della scelta per il singolare dei maestri della scuola francescana in campo gnoseologico e ontologico ha avuto poi puntuali esiti e riscontri sul piano dell'etica e della politica: non è l'universalità il punto di partenza per valutare la moralità degli atti o la validità delle istituzioni politiche, bensì la volontà deliberante del soggetto morale o del soggetto politico, la coscienza dell'individuo e la sua caratteristica peculiarità sul piano storico-esistenziale.

Una diversa concezione della natura emerge dalle tesi di Scoto e Ockham circa la creazione: anzitutto la natura non ha alcuna valenza come entità astratta. Essa sta a significare gli individui concreti che popolano il cosmo, portatori di caratteristiche che consentono alla mente di raggrupparli in specie in base a note possedute individualmente; su questa linea, che comporta l'abbandono dei dogmi aristotelici nel campo della fisica (luoghi naturali, eterogeneità dei corpi celesti, impossibilità del vuoto), la rinuncia al "principio di pienezza" (Lovejoy), per cui l'universo é tutto riempito da ogni tipo di essere possibile, si sviluppa tutta la successiva filosofia della natura, sino alla nascita del metodo scientifico moderno con Francesco Bacone, Galileo Galilei e Isacco Newton. In secondo luogo, è corretto parlare di natura specifica non ipostatizzandola come entità universale: essa esprime il possesso di identiche caratteristiche da parte di individui. E' perciò corretto parlare di atura umana, chiedendosi ad esempio quale sia il fine ultimo proprio della natura umana, ossia il fine di tutti gli esistenti sulla terra dotati di intelletto e di volontà. Degli uo-

21 L. HONNEFELDER, Ens inquantum ens. Der Begriff des Seienden als solchen als Gegenstand der Metaphysik nach der Lehre des Johannes Duns Scotus, Münster 1979, p. 254. 
mini diremo, per concludere, che la caratteristica principale, secondo Scoto e Ockham, è quella di possedere la libertà: già con la conduta l'uomo si è distinto per la libertà della volontà; la sua salvezza consegue a un altro atto di libertà dell'uomo, quello di aderire alla riapertura di credito operata dall'incarnazione, di volere liberamente seguire un tragitto che porta all'unione con Dio. La libertà della creatura non è titanismo o velleitarismo, così come la libertà radicale del Dio onnipotente non è irrazionalismo o arbitrarismo. C'è una libertà radicale, quella del Creatore, che istituisce la libertà dell'individuo, libertà certamente condizionata, ma che è il contrassegno esclusivo ed esaltante dell'uomo su tutto il creato. 